\title{
Towards a Comprehensive View of Binary and Multiple Systems
}

\author{
ALAN H. BATTEN \\ Dominion Astrophysical Observatory, Herzberg Institute of Astrophysics, \\ National Research Council of Canada, 5071 W. Saanich Road, Victoria, \\ B.C. V8X 4M6, Canada
}

\section{OPENING REMARKS}

First, I wish to express my appreciation of the honour implied by the dedication to me of the proceedings of this Colloquium. It is, of course, gratifying that colleagues should wish to make such a gesture, but I can think of many others in the field, many participants in this meeting, who are at least as worthy of a similar honour. I can only be grateful that the Scientific Organizing Committee chose me. I would also like to thank both it and the Local Organizing Committee, on your behalf as well as my own, for all the hard work that has made this Colloquium possible. I know enough of organizing meetings to guess at what has been involved in making this one run so smoothly. I have organized both IAU Colloquia and a meeting of the AAS, but I have never tried to do the two within three months of each other, as our hosts have done on this occasion!

\section{DISCUSSION}

This summary is not one of the kind that aims to mention every paper, nor shall I work through the papers I do mention in the order that they were presented. My job, I believe, is to prepare a digestible meal out of the ingredients you have brought. Some of those ingredients serve as seasoning and lose their own identity in improving the whole. Even the papers not mentioned have contributed to the meeting and may have influenced my thinking without my being consciously aware of the fact. Few cooks would necessarily serve the different dishes of a meal in the order in which the ingredients had been purchased, and it seems to me that many of the linkages between different papers on our programme cross the boundaries that necessarily divided our deliberations.

Before I came here, "complementary approaches" suggested to me primarily the combination of the results of spectroscopic and visual (or speckleinterferometric) observations. Two of the most beautiful demonstrations of the power of complementary approaches yet published, however, were based on different kinds of positional measurement. I refer to the orbits of $\alpha$ Virginis (Herbison-Evans et al. 1971) and $\beta$ Capricorni (Evans \& Fekel 1979). The positional measurements were obtained by the intensity interferometer and by occultation observations, respectively. Curiously, the first was not mentioned until nearly the end of our meeting (John Davis) and the second, I believe, has not been mentioned at all - even during Nat White's survey of occultation work. Speaking of these methods of observation reminds me that we need a general term - I suggest "resolvable binaries" - to cover all binaries for which an orbit in the plane of the sky can be determined. Spectroscopists like myself 
tend to refer to them all as "visual binaries", but that is a loose use of the term, which we should try to avoid.

The Scientific Organizing Committee clearly had a wider view of what approaches to the study of binary and multiple stars should be embraced by the term "complementary", and I think it was an inspired choice to begin with a session on the recent observational work on pre-main-sequence binaries. It was, of course, a logical place to begin, since it is as close to the beginning of binaries as we can at present reach. Nevertheless, many conferences of this kind begin with the basics, even the axiomatics of the field, whereas we were able to combine a study of origins with what are possibly the most significant observational results in our field today. As Bob Mathieu reminded us, a decade ago no orbit was known for any system that could be definitely described as "pre-main-sequence"; now there are eighteen. Just as important, surveys show that resolvable pre-main-sequence binaries are quite common. All of the several independent groups reporting here agreed that the incidence of binaries among pre-main-sequence objects is at least equal to that amongst solar-type stars. Andrea Ghez believes that $T$ Tauri stars have twice the main-sequence binary frequency, but Helmut Abt suggested that this might be partly a result of the different techniques used for detection of binaries. The suggestion was even made that all stars are formed as binaries (Christoph Leinert) but others (Michal Simon and Hans Zinnecker) found evidence for different pre-main-sequence binary frequencies in different regions of the Galaxy (see also the poster paper by Wen Ping Chen and John Graham) perhaps reflecting different conditions in the local interstellar medium. More study of these variations is needed.

Andrea Ghez also suggested that initially many triple stars are formed, but that they are unstable and break up before the components reach the main sequence. She also thought that separations tended to evolve - an idea echoed in a later session by Arcadio Poveda for somewhat more evolved systems. He found that separations of wide binaries tend to decrease with age and attributed this to the effect of the break-up of the associations to which they belong.

If changes occur in the pre-main-sequence stage, this raises an important problem to which Dan Popper drew our attention during the general discussion. Is the study of distributions of quantities such as the mass-ratio, the separation, or the degree of multiplicity telling us anything about the origin of binaries if the distribution functions are being seriously modified in the early stages of the life of binaries? People were reluctant to discuss this point, but we do have to think about it. Bob Mathieu, for instance, said that the characteristics of the eccentricity distribution are fixed before the component stars reach the main sequence, and he elaborated the point in a poster paper. At short periods, circularization is very rapid, while at longer periods eccentricities seem randomly distributed between values of about 0.1 and 0.8 . He presented a rough theory of disk-star interaction to explain this result. Now eccentricities greater than 0.8 , or even $\mathbf{0 . 9}$, are known. Though few in number, they are well determined. Michel Mayor reported one of 0.975 , exceeding the largest previously known (0.96). Do we require a special mechanism - possibly a third body - to produce these high eccentricities, or do binary orbits form that way? Alan Boss seemed to think that any initial eccentricity is possible. Mathieu's sample is still small and very high eccentricities may be at the tail-end of a frequency distribution and therefore rather rare. 
Just over thirty years ago, at the fist IAU Colloquium on double stars, Finsen dismissed the period-eccentricity relation (at least for visual binaries in the classical sense) as an artifact of observational selection (Lippincott 1962). Jean Dommanget disagreed with him then, as he reminded us during our own discussions. When we look at the whole population of binaries, we can scarcely believe any more that the period-eccentricity relation is wholly caused by observational selection. Circularization of very short-period orbits now seems well established, and we believe that we understand in astrophysical terms why it takes place in roughly the period-range, and the times, that we observe. This seems to me a genuine advance in knowledge and is unlikely to prove illusory. Let us not forget, however, the insight of thirty years ago: that observational selection may be contributing to the relation that we observe. I shall come back to the question of distributions of binary properties later, because I believe that this Colloquium produced other insights.

I shall jump now to the session on the theory of binary formation since, in my mind, that is genuinely complementary to this new observational work. It is no coincidence that observational progress on pre-main-sequence systems and theoretical progress on the formation of binaries have gone hand-in-hand during the last decade. As is so often the case they both spring from the application of a new observing technique - in this instance infra-red detection. So the observers and theoreticians have stimulated each other. Cathie Clarke dismissed the capture of stars by protostellar disks as a means of producing binaries in large clusters. Paradoxically, it may work in small sub-clusters. She suggested that the theoretical mass-ratio distribution would be such that brown dwarfs were unlikely to be found coupled with solar-mass stars. (Observationally, there seemed little agreement on brown dwarfs: some said none were known, or at least none definitely, but David Latham makes a good case for HD 114762 and Michel Mayor has found more low secondary mass-functions than he believes can be accounted for by assuming low inclinations.)

Fred Adams told us theory has difficulty in producing binaries at all, but Alan Boss appeared confident that fragmentation before the "pre-main-sequence stage" could produce a wide range of binaries with all possible mass-ratios, all possible orbital eccentricities, and separations between 1 A.U. and 1,000 A.U. He found it difficult, however, to predict the mass-ratio distribution. A particular example of binary production by fragmentation was given in a poster paper by Ian Bonnell and Pierre Bastien. The question was raised "what can fragmentation not predict?" and the answer, of course, is a really close binary with a period of a few days or less. As Mathieu's results show, such binaries exist before their components reach the main-sequence. A few months ago, I was discussing this same point at IAU Symposium 151 in Córdoba, Argentina. Citing a paper by Lebovitz (1989), I argued that, since many assumptions were made in any theoretical treatment of fission, failure to obtain a binary by such means does not necessarily invalidate the concept of fission rather than one or more of the additional assumptions. Two theoreticians in the audience approached me privately (and independently) afterwards. One assured me that fission is completely impossible, while the other expressed total agreement with what I had said! Faced with these divergent opinions from two people, each more qualified than I am to discuss the matter, I persist in suspending judgement on the question of fission. 
To return to observational papers, Helmut Abt with D.W. Willmarth emphasized that the mass-ratio distribution is different for B-type binaries from that for solar-type stars (a point also made by Michel Mayor and, for Praesepe, in poster paper by Pavel Kroupa and Chris Tout). Abt saw a possible explanation for this in the theory (Aarseth and Hills 1972) that early-type binaries are formed by multiple captures in a cluster - the end product being a "close" binary containing, usually, the two most massive stars in the original cluster. Poveda objected that this process is too slow: I agree, and add that Aarseth and Hills used the term "close" in a rather different sense from the way most of us would, namely purely relatively to the separations of the initial pairs. Their "close" pair would have quite a long period.

Only two invited papers were completely devoted to multiple systems an observational one by Frank Fekel and theoretical one by Bob Harrington. Several specific multiples were discussed in poster papers. Fekel has made the study of multiple systems very much his own and showed us a good example of a spectroscopic quintuple. We also know sextuple systems, but not - as far as I am aware - any higher multiples. Fekel emphasized the increasing difficulty of detecting such more complex systems, and opined that there probably is a limit to the number of stars one can have in a gravitationally bound system. I agree with him, and would suggest that limit is close to ten. Harrington pointed out that the ambiguity in the mutual inclinations of the two orbits of a triple system can sometimes be settled on dynamical grounds. He also said that the chief factor determining the stability of a triple is the ratio of the two orbital periods. I understood that the borderline ratio is around 5 , so $\lambda$ Tauri, with a ratio of 8 - the smallest known, is stable. So also is $0 \Sigma 341$ with a ratio of between 7000 and 8000 , even though every 20 years the third body comes exceedingly close to the close pair.

Many speakers discussed the "gap" between spectroscopic and (classical) visual binaries and how photoelectric radial-velocity measurement and speckle interferometry together are helping us to fill it. Antoine Duquennoy's graph demonstrating a continuity in the period distribution was shown several times, while Roger Griffin made the same point with a set of histograms. We seem to be converging towards agreement that there is one underlying population of binaries and that the old distinction between spectroscopic ("close") and visual ("wide") is indeed an artifact of our methods of observation. One paper by Tsevi Mazeh (with D. Goldberg) - must give us pause, however, before we jump to that conclusion. Presenting a new algorithm for deriving the mass-ratio distribution of single-spectrum binaries (which, I believe, contains an important new insight) they find that the mass-ratio distribution differs for close binaries and wide binaries. If they are right, the period distribution is telling us one thing and the mass-ratio distribution another. I would like to believe the periods which, indeed are the more directly observed quantities - but I know that we must study the mass-ratios still more before we can be sure.

I promised to return to the question of distributions. It seems to me that an underlying theme of this Colloquium has been that where we once thought of unique relations (period-eccentricity) or distributions (mass-ratio, major axis or separation), we are coming to understand that we should think of plural relations (or distributions). The period-eccentricity relation certainly changes with age, because the upper limit for circularization increases. Mass-ratio distribu- 
tions may also depend on age (results on this were presented by Duquennoy and Latham at meetings of Commission 30 in Buenos Aires), primary mass (this Colloquium, as already mentioned) and possibly with factors related to the location of sub-populations of binaries within the Galaxy. Separations, as we saw above, may also be age-dependent. Perhaps we should be looking at distributions of these quantities in different groups - clusters, Populations I and II (there really are Population II binaries, that now seems clear from several sources), binaries in different ranges in total mass, etc. ). One might recall that although the initial plotting of the Hertzsprung-Russell diagram was a tremendous step forward in observational astrophysics, not until we began to plot its equivalent, the colourmagnitude diagram, for individual clusters, did we really learn the lessons it had to teach us about stellar evolution. The difficulties, of course, in plotting, say, a mass-ratio distribution for an individual cluster are the familiar ones of small sample size and incompleteness, with their consequence of observational selection. But modern methods of observation are helping us to minimize these. Consider, for example, the very thorough (but probably still incomplete) knowledge we now have of spectroscopic binaries in the Hyades (Griffin et al. 1985).

Popper (1980) and Anderson (1991) have both lamented the relatively small contribution made to precise masses by visual binaries. In the absence of radialvelocity observations, the limit on accuracy is set by the uncertainty in the parallax, the cube of which, of course, determines the total mass. Both Wulff Heintz and Bill van Altena illustrated the difficulties that this is causing - at least until HIPPARCOS parallaxes become available. Heintz mentioned that several factors are improving the determination of parallaxes from radial velocities for resolvable binaries. Indeed we can now get very good total masses for such binaries, even without going to the very precise velocity determinations advocated by Gordon Walker for the detection of brown dwarfs or other planetary systems. Our weakness is in getting good mass-ratios (and therefore individual masses). One reason for this is that we spectroscopists often choose deliberately to observe resolvable binaries (but remember that, as the spectrograph slit sees them, they are not resolved!) with eccentric orbits and longitudes of periastron close to $0^{\circ}$ or $180^{\circ}$. This means that at one node (near periastron) we have a very large velocity difference, and the spectra are easily resolved, while at the other the velocity difference is too small even for modern techniques of observation and reduction. We then have a special case of the sort of correlation between orbital elements that Chris Morbey was talking of, and it becomes difficult to separate the elements $V_{0}, K_{1}$, and $K_{2}$, so that $K_{2} / K_{1}$ may be very uncertain. There is a challenge here both to instrumentalists and to the writers of reduction software.

Space astrometry is obviously going to be a very important complementary approach. Both HIPPARCOS and Hubble have had their misfortunes, but each is sending results of which we had foretastes at this meeting. The recovery of HIPPARCOS from its nearly disastrous launch will surely, one day, be seen as one of the greatest triumphs in space exploration. Paul Couteau told us that the discovery of new binaries would continue, but by modern means. Almost immediately he was proved right by the speakers associated with HIPPACAROS, who told us of thousands of discoveries. In our field, space research is still a promise, the necessary long-term research is likely to be ground-based for a long time to come. I was particularly impressed, however, by the potential for measuring magnitude differences from space for close pairs. The measurement 
of such differences, like that of mass-ratios, is still one of our weak points. As I hinted above, many of the most interesting pairs, even if they are relatively easy objects for traditional visual observers, are not resolved on the spectrograph slit: they are effectively "spectroscopic binaries" from the point of view of determining separately the spectra and luminosities of their components. It is, in fact, notoriously difficult to separate the contributions of two stars to a combined spectrum, except in the special case of a totally eclipsing system, when one spectrum can be observed for a short time. Ken Wright, in Victoria, pioneered a long time ago the separation of the component spectra in supergiant eclipsing systems (such as 31 Cygni) and Roger Griffin showed us a beautiful example of how he and Elizabeth Griffin have been able to refine the process with digital subtraction of the spectra. This method can be applied to only a small proportion however, and it is encouraging that, either from space, or - as some poster papers lead us to hope from ground-based CCD or speckle photometry - we can expect to obtain vastly increased numbers of measures of magnitude and colour differences.

In general discussion, Dan Popper suggested that we had relatively neglected (in this Colloquium) the photometry of eclipsing binaries as one complementary approach. This neglect is, of course, a consequence of geometry. We are mostly concerned with binaries whose components are too widely separated for there to be much likelihood of eclipses. I estimate that about one in 2000 binaries containing two solar-type stars separated by $20 \mathrm{~A}$.U. would show even partial eclipses. The permissible range of inclinations is very narrow, and even a "rectilinear" orbit is no guarantee that the system will fall within the range. For the most part, eclipsing resolvable binaries will be systems containing supergiant, or at least giant stars. Before the advent of speckle interferometry, there were - as I recall - only two systems for which photometric, spectroscopic and astrometric observations of their orbits had been made. Those systems, $\epsilon$ Aurigae and VV Cephei, were both pathological. The number is increasing now, but only slowly, and we have not yet found any of the one in 2000 main-sequence systems!

Our final sessions concerned the instrumentation that is making all these impressive advances possible. Speckle interferometry, whether at CHARA, in the former Soviet Union (described for us by Yuri Balega and Andrei Tokovinin), or in France has become the dominant method of resolving binaries. One poster paper even described how amateurs may make speckle observations - surely the sign of maturity of a new technique! I was initially surprised, therefore, when Bill Hartkopf suggested that the days of speckle techniques on the largest telescopes were already numbered - but then it became clear that this was because of the development of several long-baseline optical interferometers, not only in the U.S. but in Europe and, perhaps most importantly, in Australia. Bill Hartkopf also demonstrated that southern-hemisphere observations still lag behind those in the north, and this gives special significance to SUSI, the interferometer that John Davis is even now commissioning in Sydney.

There are thus plenty of reasons for us to look forward to still more significant results in our field in the near future, but I want to end with a quick backward glance to that colloquium in Berkeley, thirty years ago, to which both Roger Griffin and I, quite independently, have already referred. Several of the giants of our field were present there, and one supergiant - Ejnar Hertzsprung, 
who wanted that Colloquium to send a letter to the heads of all graduate schools to the effect that "every student of practical astronomy" should be compelled to spend at least some time in the visual measurement of double stars, so that those in whom the talent was discovered could be encouraged to spend their lives in doing this necessary work. Hertzsprung was nearly ninety years old at the time, and perhaps did not fully realize the practical limitations, even in those days, within which the heads of graduate schools had to work. He had to accept a rather watered-down resolution that the other participants would agree to, but the text of his proposed letter was printed in the proceedings. Now that we have impersonal methods of making the necessary measurements, Hertzsprung's proposal is no longer needed. He is also supposed to have said - and the proceedings hint at something very like this - that all astronomers should spend some of their time making observations that would be of use only to future generations. This is something that all observers of long-period resolvable binaries do, and you are here because you acknowledge the importance of observing for posterity. We are the only people who can record the sky as it is in 1992 . Even with today's multitude of astronomers, we can hardly hope to record all aspects of the contemporary sky, but we should not restrict ourselves to those aspects of it that are fashionable in our generation. Unfortunately, the criteria for funding, for allocation of telescope time, even for promotion and tenure, all place a premium on the value of tackling fashionable problems in a way that gives quick results. It is difficult, although as the existence of CHARA fortunately shows, not impossible to get funding for long-term work that can not lead to quick publication. As David Latham reminded us, even the selection of standards requires many observations over many years, with very little in the way of publishable return. It almost seems as if a standard begins to vary at the very moment that is adopted! Important as some of the results coming out of this conference may be, the encouragement it gives to you, and the example it sets to others, may be even more important for the long-term health of astronomy.

\section{REFERENCES}

Aarseth, S.J \& Hills, J.G. 1972, $A \mathcal{B} A, 21,255$

Andersen, J. 1991, $A \mathcal{E} A R, 3,91$

Evans, D.S. \& Fekel, F.C. 1972, ApJ, 228, 497

Griffin, R.F. Gunn, J.E., Zimmerman, B.A., \& Griffin, R.E.M. 1985, AJ, 90, 609

Herbison-Evans D., Brown, R. Hanbury, Davis, J., \& Allen, L.R. 1971, MNRAS, 151, 161

Lebovitz, N.R. 1989, in Highlights of Astronomy, ed. D. McNally, (Dordrecht, Kluwer), 8, 129

Lippincott, S.L. 1962, PASP, 74, 5

Popper, D.M. 1980, ARA\&A, 18, 115 